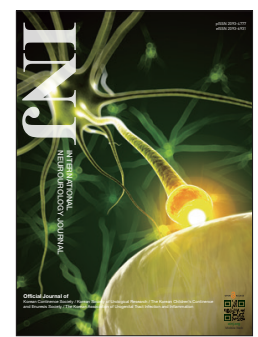

\title{
Angiopoietin-1 and Angiopoietin-2 Expression Imbalance Influence in Early Period After Subarachnoid Hemorrhage
}

\author{
Hua Gu*, Zhen-Hai Fei*, Yi-Qi Wang, Jian-Guo Yang, Chao-Hui Zhao, Yong Cai, Xing-Ming Zhong \\ Department of Neurosurgery, The First Affiliated Hospital of Huzhou Teachers College, Huzhou, China
}

Purpose: Microvascular endothelial integrity is important for maintaining the blood-brain barrier (BBB). However, subarachnoid hemorrhage (SAH) disrupts this integrity, making the BBB dysfunctional — an important pathophysiological change after SAH. Angiopoietin-1 (Ang-1) and angiopoietin-2 (Ang-2) regulate microvascular permeability by balancing each other's expression.

Methods: This study investigated the dynamics of Ang-1 and Ang-2 expression after SAH and the protective effect of Ang-1 on BBB functioning using an endovascular puncture model of rat SAH. The Ang-1 and Ang-2 expression in brain tissue was determined by immunohistochemistry. In addition, Western blotting was used to estimate Ang- 1 and Ang- 2 concentration and to compare them at 6-72 hours post-SAH cortex and hippocampus. Evans blue viability assay was used to evaluate BBB permeability, and neurological testing was implemented to evaluate neurological impairment during SAH.

Results: It was found that following SAH, Ang-1 expression decreases and Ang-2 expression increases in the cortex, hippocampus, and microvessels. The Ang-1/Ang-2 ratio decreased as quickly as 6 hours after SAH and reached its lowest 1 day after SAH. Finally, it was found that exogenous Ang-1 reduces SAH-associated BBB leakage and improves neurological function in post-SAH rats.

Conclusions: Our findings suggest that the equilibrium between Ang-1 and Ang-2 is broken in a period shortly after SAH, and the treatment of exogenous Ang-1 injection alleviates neurological dysfunctions through decreasing BBB destruction.

Keywords: Angiopoietin-1; Angiopoietin-2; Subarachnoid Hemorrhage; Blood-Brain Barrier; Brain Injuries

- Fund Support: The National Natural Science Foundation of China provided financial support (No. 30870844). The sponsor had no role in the design or conduct of this research.

- Research Ethics: All animal procedures were reviewed and approved by the Laboratory Animal Center of Xi'an Jiaotong University, which were formulated by the Ministry of Science and Technology of the People's Republic of China. All procedures performed in studies involving animals were in accordance with the ethical standards of the institution or practice at which the studies were conducted.

- Conflict of Interest: No potential conflict of interest relevant to this article was reported.

\section{INTRODUCTION}

Subarachnoid hemorrhage (SAH) is one of the most common hemorrhagic cerebrovascular diseases associated with high morbidity and mortality. About $75 \%-80 \%$ of SAHs are caused by the rupture of intracranial aneurysms [1]. Early brain injury (EBI) is an early and important pathophysiological consequence of SAH [2]. The disruption of the structural integrity of the blood-brain barrier (BBB) plays an essential role in EBI and has been found within hours after aneurysmal SAH; it causes a

Corresponding author: Xing-Ming Zhong (iD http://orcid.org/0000-0002-7906-7843 Department of Neurosurgery, the First Affiliated Hospital of Huzhou Teachers College, No. 158 Guangchanghou Road, Huzhou, Zhejiang Province 313000, China E-mail: 15209203424@163.com / Tel: +86-13967259575 / Fax: +86-5722039316 ${ }^{*} \mathrm{Hua} \mathrm{Gu}$ and Zhen-Hai Fei contributed equally to this study as co-first authors. Submitted: August 13, 2016 / Accepted: October 3, 2016 
series of pathologic changes such as brain edema, thrombosis, inflammation and abnormal cerebral metabolism [3-5]. Therefore, microvascular dysfunction and BBB destruction are considered to be the most feasible targets of SAH treatment [6].

Endothelial cell stability is crucial for BBB integrity. These cells secrete angiopoietins - a protein family of endothelial growth factors that promotes angiogenesis. Angiopoietins regulate vascular homeostasis by binding to and activating the endothelial Tie receptor tyrosine kinases [7]. Angiopoietin-1 (Ang-1) binds to and phosphorylates Tie-2 receptor. Ang-1 plays an important role in vascular-stabilization, endothelial cell maturation, adhesion, migration, and survival, and has antiinflammatory effects [8]. Angiopoietin-2 (Ang-2) also binds to Tie-2 but as an antagonist of Ang-1. Ang-2 mediates endothelial cell death, disrupts vascularization, and increases vascular permeability, and has pro-inflammatory effects $[9,10]$. In the central nervous system, the high level of Ang- 1 and low level of Ang- 2 constitute a dynamic balance which is required to maintain BBB integrity by regulating endothelial Tie-2 phosphorylation level [11]. Recent in vitro studies have shown that Ang-1 can reduce the destruction of the BBB [12]. Lower Ang-1 and higher Ang-2 levels have been found in the serum of SAH patients. The studies also associated a low Ang-1 to Ang-2 ratio with cerebral vasospasm (CVS) and delayed cerebral ischemia (DCI) prospectively [13]. All of this evidence suggests that a lower Ang-1 to Ang-2 ratio is a key process responsible for post-SAH BBB destabilization. Therefore, it has become very critical to understand the pathophysiological effects and mechanisms related to Ang-1 and Ang-2 activities and dynamics in the early course of SAH.

This study hypothesizes that the Ang-1 and Ang-2 equilibrium is broken in the early period of SAH, and that the restoration of this equilibrium through Ang-1 treatment can improve BBB stability and neurological functioning after SAH.

\section{MATERIALS AND METHODS}

\section{Animals}

A total of 150 male Sprague-Dawley rats weighing 250-300 g were purchased from the Laboratory Animal Center of Xian Jiaotong University and used for the study. The rats were housed in 12-hour light and dark cycles in a temperature and humidity- controlled environment, and they had free access to food and water. The rats were housed and handled in strict accordance with National Institutes of Health's and Xian Jiaotong
University's research animal care and use guidelines. All animal procedures were reviewed and approved by the Laboratory Animal Center of Xian Jiaotong University, which were formulated by the Ministry of Science and Technology of the People's Republic of China.

\section{Rat Endovascular Puncture Model}

$\mathrm{SAH}$ was induced by endovascular perforation technique as described by Bederson et al. [14]. A sharpened Prolene 3.0 suture was introduced into the right external carotid artery and was advanced through the internal carotid artery. The common carotid artery was kept clamped near the junction of external and internal carotid arteries. The suture was further advanced into the intracranial internal carotid artery until resistance was felt and then it was pushed $3 \mathrm{~mm}$ further into the internal carotid artery near the middle cerebral artery bifurcation. The duration of endovascular occlusion was between 30 seconds and 2 minutes. Fig. 1B-E shows representative hemorrhages. The sham rats were operated on similarly; however, the bifurcation of the internal carotid artery and middle cerebral artery was not punctured, and without hemorrhages in the subarachnoid space (Fig. 1A).

In the first set of experiments, 70 rats were randomly divided into 5 groups: sham-operated $(n=20), 6$-hour post $S A H(n=10)$, 1-day post SAH $(n=20), 2$-day post $S A H(n=10)$, and 3-day post SAH $(n=10)$. All sham-operated rats were euthanized on day 1 . The SAH rats were euthanized at 6 hours. 1 day, 2 days, and 3 days after puncturing. At respective time points, 10 rats per group, including the sham-operated group, were euthanized and perfused with saline. The cerebral cortex and the hippocampus were collected from these perfused rats for Western blotting. The remaining 20 rats, 10 from 1-day post SAH group and 10 from the sham-operated group, were used for immunohistochemical staining. Their brains were washed in normal saline solution and installed overnight at room temperature in $4 \%$ paraformaldehyde in $0.01 \mathrm{M}$ phosphate buffered saline. The tissue blocks were then dehydrated with an ascending ethanol solution series, cleared with xylene, and embedded in paraffin. The paraffin blocks were cut into transverse serial sections of 10$\mu \mathrm{m}$ thickness. Next, 5 sections containing the hippocampus and cortex were randomly taken from each perfused rat and mounted on poly-L-lysine coated slides for immunohistochemical staining. 

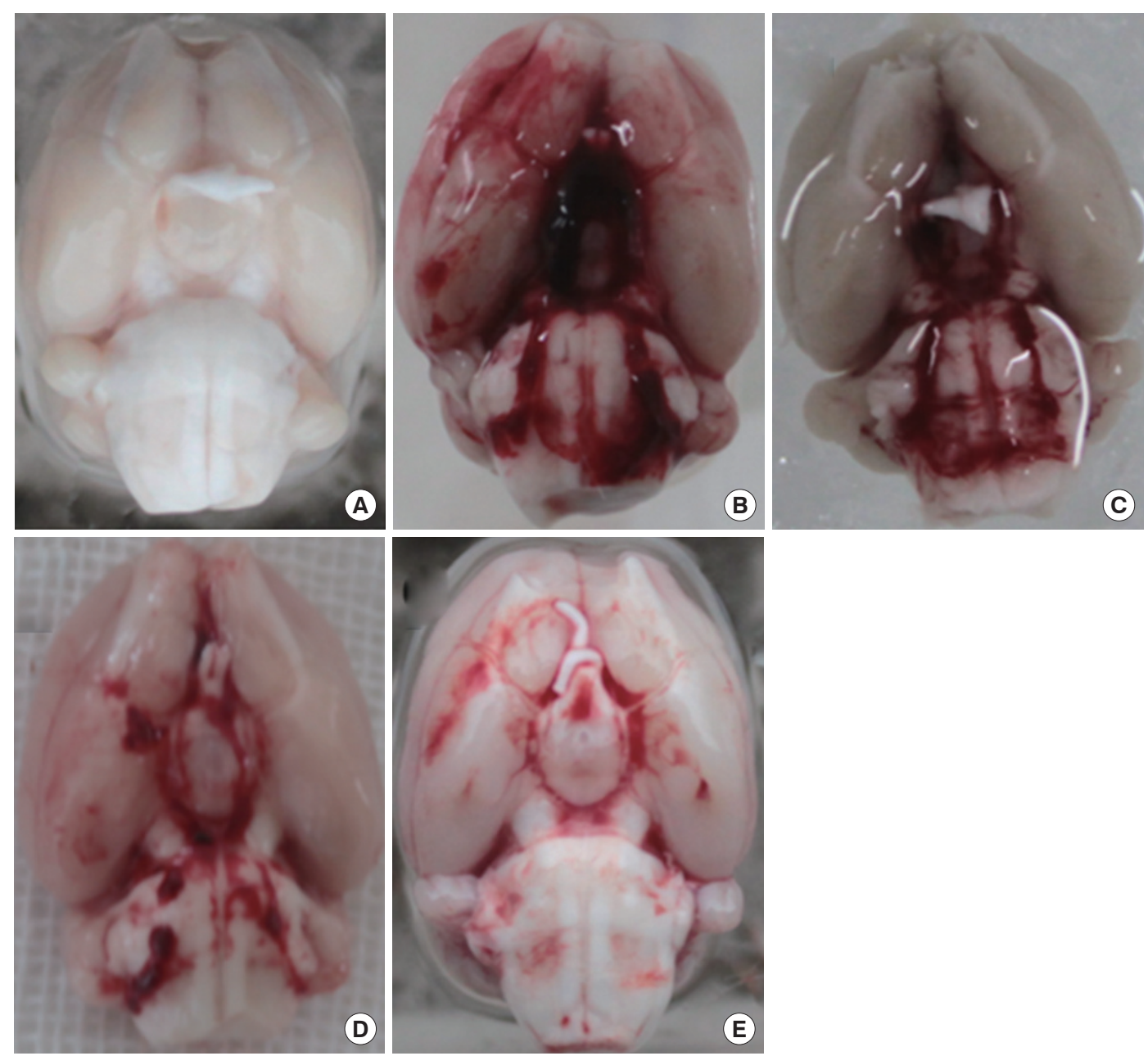

Fig. 1. The ventral brain from a sham-operated rat (A) after 6 hours (B); 1 day (C); 2 days (D); and 3 days (E) after subarachnoid hemorrhage.

\section{Immunohistochemistry}

Immunohistochemistry was performed to study Ang-1 and Ang-2 expression in post SAH hippocampuses and cortices. The sections were deparaffinized and rehydrated in graded concentrations of ethanol and finally in distilled water. The sections were incubated in 3\% hydrogen peroxide for 5 minutes to block the endogenous peroxidase activity. To retrieve the hidden epitopes of Ang-1 and Ang-2 proteins, the sections were placed in 10-mmol/L citrate buffer $(\mathrm{pH}, 6.0)$, heated in a microwave oven at $95^{\circ} \mathrm{C}$ for 30 minutes, cooled at $25^{\circ} \mathrm{C}$ for $20 \mathrm{~min}$ utes, and then rinsed in phosphate-buffered saline (PBS). The nonspecific protein binding was blocked by incubating the sections in $5 \%$ horse serum in PBS for 1 hour at $25^{\circ} \mathrm{C}$. The sections were then probed with primary antibodies (anti-Ang-1, 1:1,000, Abcam, Cambridge, MA, USA; anti-Ang-2, 1:500, Abcam) overnight at $4^{\circ} \mathrm{C}$, followed by a 15 -minute wash in PBS. The sections were then incubated in horseradish peroxidase (HRP)conjugated secondary antibody (1:500, Abgent) for 60 minutes at $25^{\circ} \mathrm{C}$. Diaminobenzidine was used as a chromogen, and hematoxylin as a counterstain.

\section{Western Blot Analysis}

Using a tissue homogenizer, the frozen tissue samples were solubilized in a RIPA buffer on ice. Sixty $\mu \mathrm{g} / \mathrm{lane}$ of protein samples were separated in a sodium dodecyl sulfate-polyacrylamide gel (10\% or $12 \%$ acrylamide gel) and electrotransferred onto polyvinylidine fluoride membranes. After 2 hours of incubation in a blocking solution (5\% nonfat milk in $20 \mathrm{mM}$ Tris$\mathrm{HCl}, 150 \mathrm{mM} \mathrm{NaCl}, 0.1 \%$ Tris-buffered saline with Tween 20 [TBST]), the membranes were blotted with primary antibodies 
against Ang-1 (1:30,000, Abcam, ab183701), Ang-2 (1:5,000, Abcam, ab155106), or b-actin (1:1,000, Beijing Biosynthesis Biotechnology, Beijing, China) overnight at $4^{\circ} \mathrm{C}$. After extensive rinsing with TBST buffer, the membranes were incubated with HRP-conjugated secondary antibodies. The antigen specific bands were detected using a chemiluminescence kit (Heliosense Biotechnologies Inc., Xiamen, China) and X-ray films (Heliosense Biotechnologies Inc.). Western blots were performed on at least 6independent experiments. After scanning the X-ray films, the density of the bands was analyzed with Quantity one software ver. 4.62 (Bio-Rad, Hercules, CA, USA).

\section{Ang-1 Peptide Treatment}

In the second set of experiments, 80 rats were randomly assigned to 4 groups: sham-operated $(n=20), S A H ~(n=20)$, SAH + vehicle $(n=20)$, and SAH+Ang- $(n=20)$. The rats were operated on to induce $\mathrm{SAH}$ as described above. Immediately after inducing $\mathrm{SAH}$, the rats were injected with Ang-1 peptide (Abcam, ab109637) dissolved in distilled water at $100 \mu \mathrm{g} / \mathrm{mL}$. The preliminary experiment demonstrated that the dose of $50 \mu \mathrm{g}$ had a more significant effect than $20 \mu \mathrm{g}$ on reducing the damage of $\mathrm{BBB}$ after $\mathrm{SAH}$, and it was a relatively safe dose for rats, so $50-\mu \mathrm{g}$ peptide was injected through the tail vein in about 3 minutes. Half of the rats from second set of experiments were euthanized and sacrificed for use of the brains for Evans Blue viability assay. The other half of the animals were subjected to neurological testing.

\section{Evans Blue Extravasation}

One day after SAH was induced, 10 rats were randomly selected from each experimental group of the second set of experiments for assessment of BBB vascular permeabilization using a modified Evans blue extravasation method. The rats were briefly anesthetized with an intraperitoneal injection of $10 \%$ chloral hydrate $(0.35 \mathrm{~mL} / \mathrm{kg})$ and infused with $37^{\circ} \mathrm{C}$. Evans blue dye $(2 \%$ in $0.9 \%$ normal saline, $4 \mathrm{~mL} / \mathrm{kg}$ ) via the right femoral vein which was injected for over 5 minutes. Two hours later, the rats were perfused with $300-\mathrm{mL}$ saline to wash out any remaining dye in the blood vessels. The brains were then removed and cut into 2-mm-thick sections. The brain slices were weighed and immersed into formamide. The BBB permeability in the brain motor cortex tissue was spectrophotometrically evaluated by measuring the absorbance of Evans blue in the supernatant (OptiMax, Molecular Devices, Sunnyvale, CA, USA) at $610 \mathrm{~nm}$.

\section{Neurological Assessment}

The rats from the second set of experiments were subjected to neurological assessment measured with a modified version of the method described by Sugawara et al. [15]. The animals were scored on spontaneous activity (score, $0-3$ ), spontaneous movements of all limbs (score, $0-3$ ), movements of forelimbs (score, $0-3$ ), climbing the wall of a wire cage (score, 1-3), reaction to touch on both sides of the trunk (score, 1-3), and response to vibrissae touch (score, 1-3). The minimum neurological score in this method is 3 and the maximum is 18 .

\section{Statistical Analysis}

For statistical analysis IBM SPSS Statistics ver. 19.0 (IBM Co., Armonk, NY, USA) was used. The data is presented as mean \pm standard deviation. Analysis of variance was used to compare multiple groups. The LSD tests were used for to compare the differences between the 2 groups. Either $\mathrm{P}<0.05$ or $\mathrm{P}<0.01$ was considered statistically significant.

\section{RESULTS}

\section{General Observations}

Twenty-five rats died during the course of the experiments, though none of the rats in the sham group died. Dead rats were excluded from further analysis. The expected decrease in postsurgical physical activity was noticed. Blood clots in the subarachnoid space 6 hours (Fig. 1B) and 1 day (Fig. 1C) post SAH were observed. These clots were gradually absorbed with time (Fig. 1D, E).

\section{Expression of Ang-1 and Ang-2 in Rat Brain After SAH}

Immunohistochemistry was used to show the expression of Ang-1 and Ang- 2 in the cerebral cortex, hippocampus, and microvascular endothelial. The distinct decrease in Ang-1 expression from the SAH group was observed in the cortex and hippocampal CA1-CA2 regions Fig. 2D-F with the sham group Fig. 2A-C. The difference in Ang-1 expression in the microvessels is shown in Fig. 2C, F. The results also show that the level of Ang-2 expression in the cortex, hippocampus, and microvessels of the SAH group (Fig. 2J-L) was higher than that in the sham group (Fig. 2G-I).

\section{Dynamics of the Ratio of Ang-1/Ang-2 Expression in the Cerebral Cortex and Hippocampus}

The western blot analysis showed significant differences of Ang1 and Ang-2 in the SAH group compared with the sham groups. 

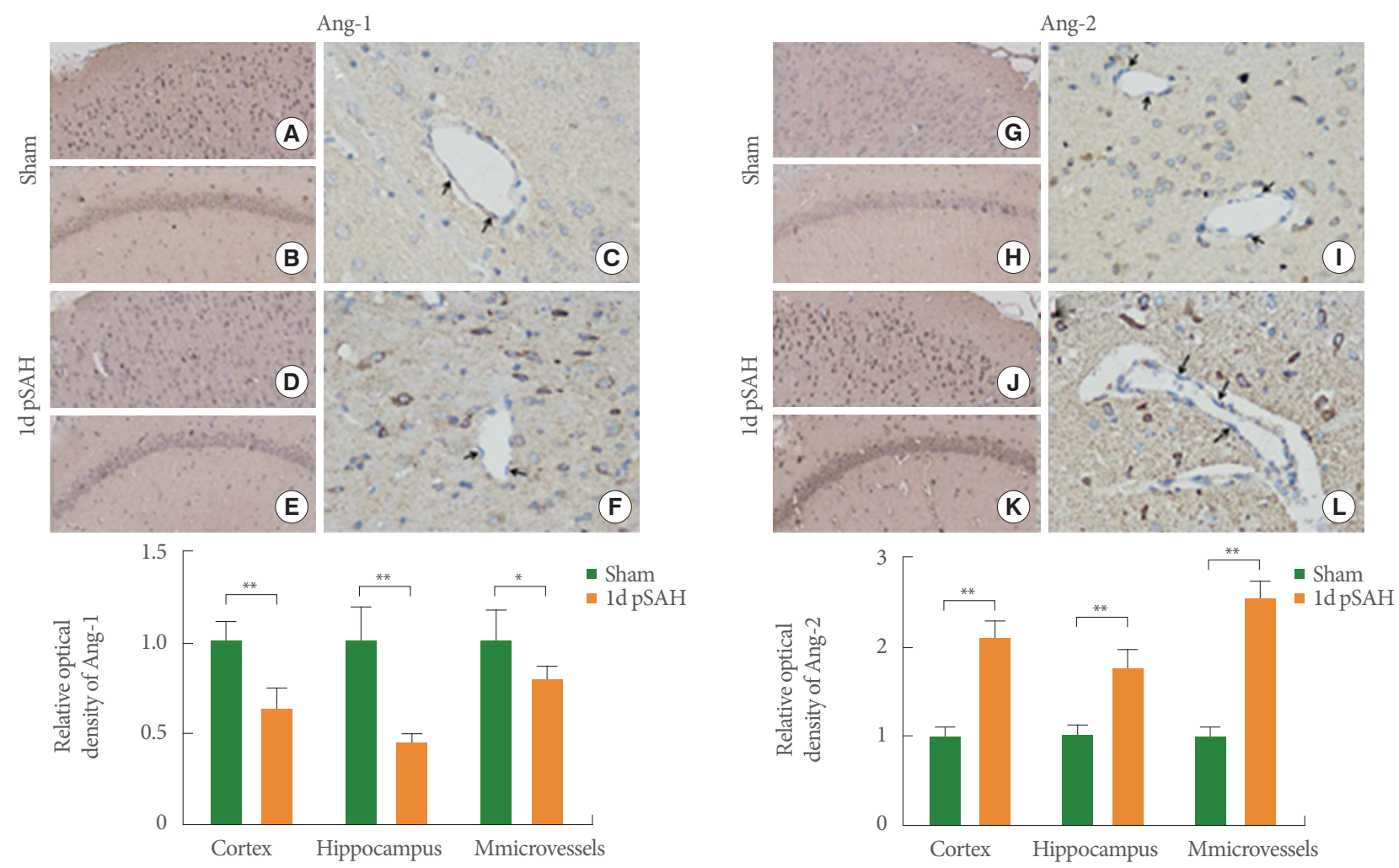

Fig. 2. Immunohistochemistry of Ang-1 (A-F) and Ang-2 (G-L): Ang-1 and Ang-2 immunolabelling in cerebral cortex (A, D, G, J), hippocampus (B, E, H, K) and microvessels (C, F, I, L) 1 day after SAH. Note decreased Ang-1 staining in SAH (D-F) rats as compared to sham $(\mathrm{A}-\mathrm{C})$ rats and increased Ang-2 staining in SAH (J-L) rats as compared to sham (G-I) rats (cortex and hippocampus, $\times 200$; microvessels, $\times 400)$. The black arrows indicate endothelial cells. Results are described as mean \pm standard deviation. Ang-1, angiopoietin-1; Ang-2, angiopoietin-2; $1 \mathrm{~d}$ pSAH, 1-day post subarachnoid hemorrhage. post $\mathrm{SAH} .{ }^{*} \mathrm{P}<0.05$. ${ }^{*} \mathrm{P}<0.01$, versus the sham group.

Two contradictory trends emerged in Ang-1 and Ang-2 expression after SAH. The Ang-1 level decreased rapidly at 6 hours and reached its lowest 1 day after $\mathrm{SAH}$ in the cortex and hippocampus (Fig. 3A, B). On the other hand, the Western blot data indicated a significant increase in Ang-2 expression in the cerebral cortex and hippocampus until the expression reached a peak at 1 day after SAH (Fig. 3A, B).The decrease of the ratio appeared at 6 hours and reached its lowest point at 1 day after SAH. The lowest value was one-twentieth less than that of the sham group in the cortex (Fig. 3C). The trend in the hippocampus was similar to that in the cortex, and the value of the low peak was only about one-ninth of that in the sham group (Fig. $3 \mathrm{D})$. For reflecting the $\mathrm{BBB}$ stabilization, it was confirmed that the Ang-1 to Ang-2 ratio was more sensitive.

\section{Exogenous Ang-1 Repairs the Disrupted Blood-Brain Barrier and Improves Neurological Scores After SAH}

The results so far suggest that the peak changes in Ang-1 and
Ang-2 expression occur at one day post SAH. Based on this, an Evans blue extravasation was done at 1 day after SAH. The results show that vehicle did not affect SAH. Less Evans blue dye leaked in the SAH+ Ang-1 group than in the SAH + vehicle group (Fig. 4A). This indicates that the Ang-1 peptide either prevents the $\mathrm{BBB}$ from disintegrating post $\mathrm{SAH}$ or helps repair it. Neurological scores were recorded at 3 days after SAH as an important and direct indicator to evaluate the neurological prognosis. By means of analyzing the results of neurophysiological testing, the rats had a significant neurological dysfunction after SAH. In addition, the therapy of Ang-1 injection succeeded in reducing neurological deficits after SAH compared with the vehicle (Fig. 4B).

\section{DISCUSSION}

$\mathrm{BBB}$ dysfunction is one of the most significant events during EBI. It is associated with a series of pathophysiological process- 

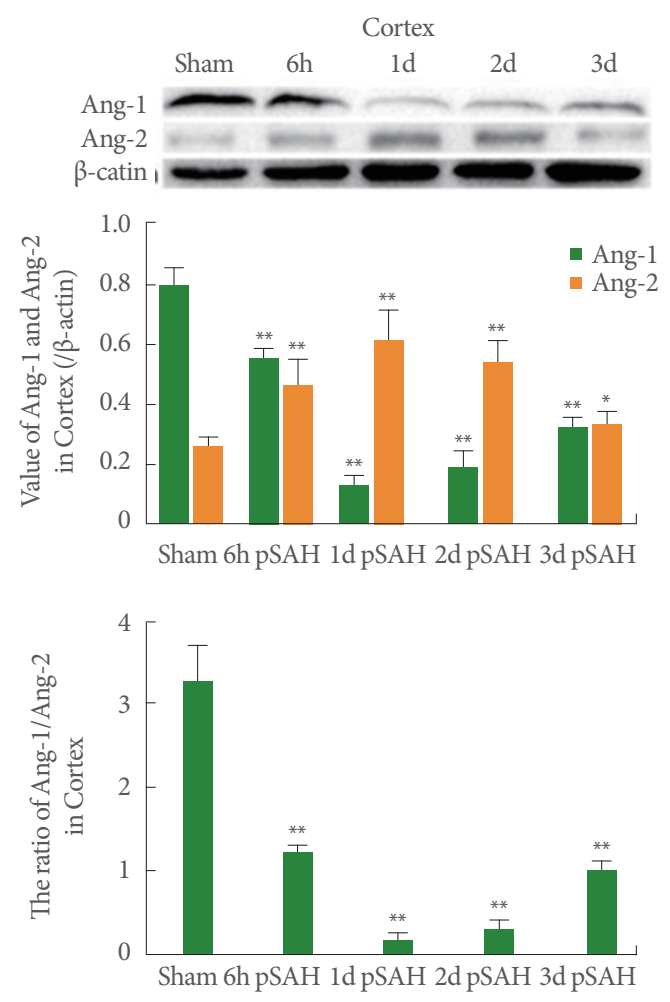

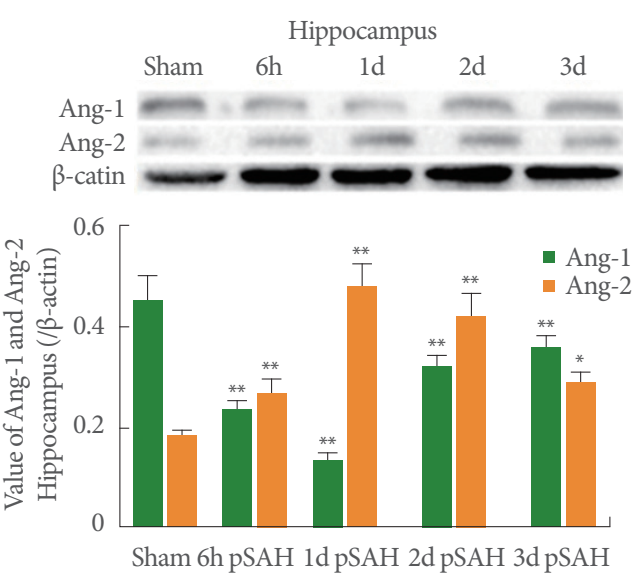

B

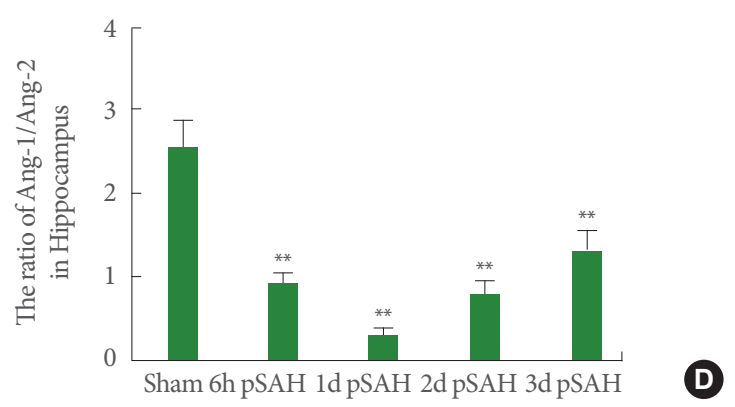

Fig. 3. Western blot and quantitative analysis of Ang-1 and Ang-2 expression: cerebral cortex (A) and the hippocampus (B), 6-hour (6h), 1-day (1d), 2-day (2d), and 3-day (3d) post SAH. The ratio of Ang-1/Ang-2 after SAH is exhibited in the cortex (C) and hippocampus (D), respectively. Results are described as mean \pm standard deviation. Ang-1, angiopoietin-1; Ang-2, angiopoietin-2; SAH, subarachnoid hemorrhage; $\mathrm{pSAH}$, post $\mathrm{SAH} .{ }^{*} \mathrm{P}<0.05 .{ }^{* *} \mathrm{P}<0.01$, versus the sham group.
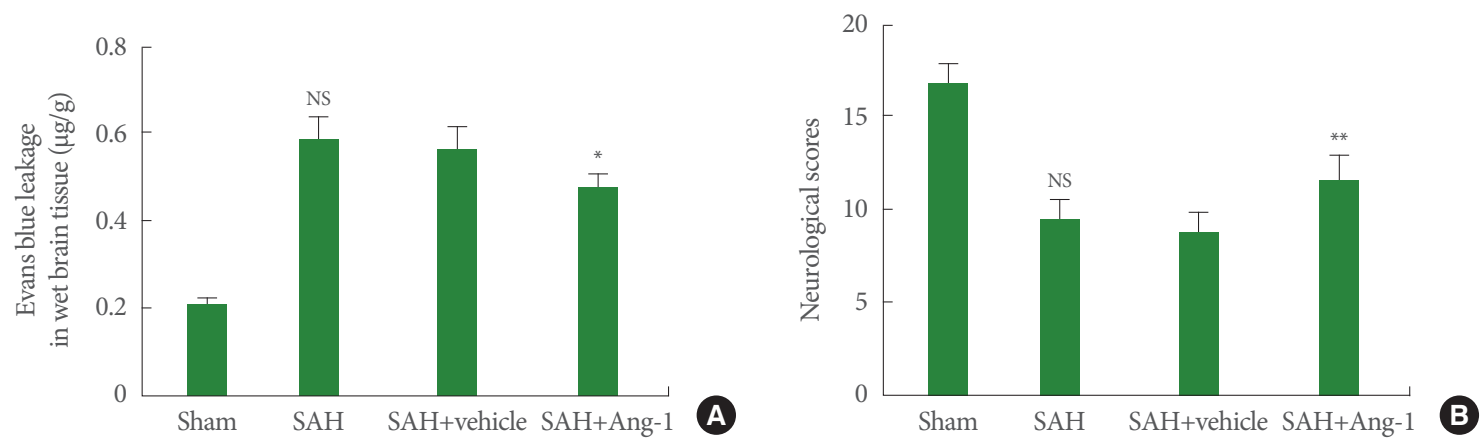

Fig. 4. Evans blue extravasation (A) and neurological scores (B): Evans blue extravasation was evaluated 1 day and neurological scores were recorded 3 days after SAH. Results are described as mean \pm standard deviation. Ang-1, angiopoietin-1; SAH, subarachnoid hemorrhage. ${ }^{*} \mathrm{P}<0.05$, ${ }^{* *} \mathrm{P}<0.01$. NS, not significant, $\mathrm{P}>0.05$ versus the $\mathrm{SAH}+$ vehicle group.

es such as microclot formation, inflammation, decreased cerebral blood flow, and CVS after SAH [5]. This study reports two important findings. First, the dynamics of Ang-1 and Ang-2 expression and imbalance after SAH were shown. Ang-1 expression started to decrease while the Ang-2 expression started to increase at 6 hours, and reached its low or high peak at 1 day after SAH. The ratio of Ang-1/Ang-2 dropped abruptly within 1 day after SAH. Then the ratio rose slightly; however, it was still lower than normal. More importantly, through the exogenous Ang-1 therapy, the BBB leakage was controlled, which could be observed by the decline of Evans blue effusion. Neurological function was improved after Ang-1 injection in SAH rats. 
According to our results, Ang-1 and Ang-2 have a crucial effect in the very early post SAH phase. This change might happen earlier than that of inflammatory factors such as tumor necrosis factor- $\alpha$ for which peak expression is at two days after SAH [16]. An in vivo study has shown that the intercellular junctions between endothelial cells became loose even before 6hours after SAH induction which results in BBB hyperpermeability [17]. This supports our hypothesis that the molecular mechanisms responsible for BBB disruption play a key role in SAH and might be a new therapeutic target for SAH-associated EBI.

A number of previous research studies about microvascular dysfunction in SAH focused on vessel related molecules like VEGF [18]. However, the angiopoietin, another crucial factor for endothelial integrality, has largely been ignored. Tie-2 is a tyrosine kinases receptor which is mainly expressed at the endothelial cell membranes. Ang-1 and Ang-2 are both the ligands of Tie-2 receptor, and compete each other to bind to Tie2 and have an opposing role in vascular stability. Ang-1 phosphorylates Tie-2 and induces vessel maturation, adhesion, migration, and survival. On the other hand, Ang-2 binds to but does not phosphorylate Tie-2, and results in vessel leakage [19]. Recent studies have found that angiopoietin/tie may regulate BBB permeability through tight junction protein mediated stabilization of cell-cell junction in brain endothelial cells [12]. Although there are many basic research studies of angiopoietins, the relationship of angiopoietins and SAH is very rarely studied. Our study reveals the functioning of Ang- 1 and Ang-2, two important endothelial vessel-related molecules in pathophysiologic processes after SAH. However, the upstream mechanism of this imbalance between Ang- 1 and Ang- 2 is not clear. For the equilibrium between Ang-1 and Ang-2 to break in 6 hours after SAH, when most of the inflammatory factors did not express, complex relationships between Ang-1/Ang-2 and inflammation exist. It can be speculated that the hemoglobin or hemoglobin degradation products in subarachnoid space may be the one of the initiating factors.

Besides disrupting the endothelial barrier, it has been proven that Ang-2 could promote neo-vascularization in conjunction with VEGF [20]. Therefore, inhibiting Ang-2 may obstruct neo-vascularization and increase the risk of delayed DCI. Ang1 is a physiological antagonist of Ang-2. However, it does not down-regulate the expression of Ang-2. This is the reason why injection of Ang-1 peptide to bring the equilibrium back during the very early phase after SAH was chosen instead of an artificial Ang-2 inhibition. Previous studies have shown that BBB permeability increased in the very early period after SAH [17]. To keep the early pathophysiological process intact, the time point of treatment was set at 0 minute after the operation.

There are 2 main models of SAH for in vivo studies: the endovascular perforation model and the double hemorrhage model. The first model has higher mortality than the second one. This study, however, chose the endovascular perforation model because it is similar to human SAH patients by simulating the rupture of intracranial aneurysms and induces more severe pathophysiological changes [15,21].

In conclusion, this study shows that the Ang-1 and Ang-2 equilibrium breaks very early after SAH induction, and exogenous Ang-1 can improve the BBB integrality and neurological impairment after SAH. The protective effect of Ang-1 in restoring angiopoietin balance could be a potential therapeutic strategy to prevent EBI for SAH patients.

\section{REFERENCES}

1. Suarez JI, Tarr RW, Selman WR. Aneurysmal subarachnoid hemorrhage. N Engl J Med 2006;354:387-96.

2. Sehba FA, Hou J, Pluta RM, Zhang JH. The importance of early brain injury after subarachnoid hemorrhage. Prog Neurobiol 2012;97:14-37.

3. Ostrowski RP, Colohan AR, Zhang JH. Molecular mechanisms of early brain injury after subarachnoid hemorrhage. Neurol Res 2006;28:399-414.

4. Pisapia JM, Xu X, Kelly J, Yeung J, Carrion G, Tong H, et al. Microthrombosis after experimental subarachnoid hemorrhage: time course and effect of red blood cell-bound thrombin-activated prourokinase and clazosentan. Exp Neurol 2012;233:357-63.

5. Song JN, Chen H, Zhang M, Zhao YL, Ma XD. Dynamic change in cerebral microcirculation and focal cerebral metabolism in experimental subarachnoid hemorrhage in rabbits. Metab Brain Dis 2013;28:33-43.

6. Egashira Y, Zhao H, Hua Y, Keep RF, Xi G. White matter injury after subarachnoid hemorrhage: role of blood-brain barrier disruption and matrix metalloproteinase-9. Stroke 2015;46:2909-15.

7. Augustin HG, Koh GY, Thurston G, Alitalo K. Control of vascular morphogenesis and homeostasis through the angiopoietin-Tie system. Nat Rev Mol Cell Biol 2009;10:165-77.

8. Brindle NP, Saharinen P, Alitalo K. Signaling and functions of angiopoietin-1 in vascular protection. Circ Res 2006;98:1014-23.

9. Daly C, Eichten A, Castanaro C, Pasnikowski E, Adler A, Lalani AS, et al. Angiopoietin-2 functions as a Tie2 agonist in tumor 
models, where it limits the effects of VEGF inhibition. Cancer Res 2013;73:108-18.

10. Maisonpierre PC, Suri C, Jones PF, Bartunkova S, Wiegand SJ, Radziejewski C, et al. Angiopoietin-2, a natural antagonist for Tie2 that disrupts in vivo angiogenesis. Science 1997;277:55-60.

11. Nourhaghighi N, Teichert-Kuliszewska K, Davis J, Stewart DJ, Nag S. Altered expression of angiopoietins during blood-brain barrier breakdown and angiogenesis. Lab Invest 2003;83:1211-22.

12. Siddiqui MR, Mayanil CS, Kim KS, Tomita T. Angiopoietin-1 regulates brain endothelial permeability through PTPN-2 mediated tyrosine dephosphorylation of occludin. PLoS One 2015;10:e0130857.

13. Fischer M, Broessner G, Dietmann A, Beer R, Helbok R, Pfausler B, et al. Angiopoietin-1 is associated with cerebral vasospasm and delayed cerebral ischemia in subarachnoid hemorrhage. BMC Neurol 2011;11:59.

14. Bederson JB, Germano IM, Guarino L. Cortical blood flow and cerebral perfusion pressure in a new noncraniotomy model of subarachnoid hemorrhage in the rat. Stroke 1995;26:1086-91.

15. Sugawara T, Ayer R, Jadhav V, Zhang JH. A new grading system evaluating bleeding scale in filament perforation subarachnoid hemorrhage rat model. J Neurosci Methods 2008;167:327-34.

16. Zhang BF, Song JN, Ma XD, Zhao YL, Liu ZW, Li Y, et al. Etanercept alleviates early brain injury following experimental subarachnoid hemorrhage and the possible role of tumor necrosis factor- $\alpha$ and cJun N-terminal kinase pathway. Neurochem Res 2015;40:591-9.

17. Li Z, Liang G, Ma T, Li J, Wang P, Liu L, et al. Blood-brain barrier permeability change and regulation mechanism after subarachnoid hemorrhage. Metab Brain Dis 2015;30:597-603.

18. Liu L, Fujimoto M, Kawakita F, Ichikawa N, Suzuki H. Vascular endothelial growth factor in brain edema formation after subarachnoid hemorrhage. Acta Neurochir Suppl 2016;121:173-7.

19. Hansen TM, Singh H, Tahir TA, Brindle NP. Effects of angiopoietins- 1 and -2 on the receptor tyrosine kinase Tie 2 are differentially regulated at the endothelial cell surface. Cell Signal 2010;22:527-32.

20. Fagiani E, Christofori G. Angiopoietins in angiogenesis. Cancer Lett 2013;328:18-26.

21. Lee JY, Sagher O, Keep R, Hua Y, Xi G. Comparison of experimental rat models of early brain injury after subarachnoid hemorrhage. Neurosurgery 2009;65:331-43. 